\section{New Physical Sign of Acute Deep Vein} Thrombosis

SIR,-We would like to draw the attention of your readers to the following two cases with an unusual and hitherto unreported sign of extensive deep vein thrombosis.

Case 1. A 54-year-old man presented to his doctor in January 1973 with pain in his left lower limb associated with inability to extend the knees fully. An analgesic was prescribed since the leg appeared normal apart from a $5^{\circ}$ flexion deformity of the knee. After two days erythema and tenderness developed along the medial aspect of the thigh. He failed to respond to an antibiotic and bed rest, the thigh became swollen, and he was referred to hospital. On examination the pulse rate was $72 / \mathrm{min}$ and temperature $36.7^{\circ} \mathrm{C}$. There was erythema along the medial aspect of the left thigh and marked swelling confined to the thigh was present. The knee was flexed to $20^{\circ}$ short of full extension and the patient was unable to extend it beyond this point. The peripheral pulses were normal. An ascending phlebogram showed complete occlusion of the deep veins of the leg from the calf to the common femoral. The long saphenous vein was patent and there was a partly occlusive thrombus in the external iliac vein. Pulmonary perfusion scan and chest $x$-ray were both normal. There was no evidence of bone or joint pathology on $x$-ray of the knee. The patient was treated with intravenous heparin and leg elevation. The swelling and erythema subsided rapidly and within $\mathbf{4 8}$ hours he was able to extend the knee fully.

Case 2. A 46-year-old man was admitted with a five-day history of severe pain and swelling in the left leg. He noted that within hours of the onset of pain in the calf he was unable fully to extend the knee. He was treated at home with analgesics but when he failed to improve after a few days he was referred to hospital. On examination his pulse was $90 / \mathrm{min}$ and temperature $37^{\circ} \mathrm{C}$. The left leg was a dusky colour and was very swollen. The knee was flexed to $25^{\circ}$ short of full extension and the patient was unable to extend it beyond this. The long and short saphenous veins were both palpably thrombosed. Abdominal and rectal examintion revealed no abnormality and there was no lymphadenopathy. Ascending phlebography showed extensive venous occlusion involving all the deep veins of the lower limb, the long and short saphenous vein, and the external iliac vein. Chest $x$-ray showed a raised left hemidiaphragm and pleural effusion. Pulmonary perfusion scan showed a segmental perfusion defect in the left mid-zone compatible with a diagnosis of pulmonary embolism. There was no evidence of bone or joint pathology on $x$-ray examination of the knee. The patient was treated with intravenous heparin infusion and leg elevation. The swelling subsided gradually during the next five days, but it was two weeks before the patient could fully extend the knee.

Loss of full extension of the knee, coming on rapidly, is almost always due to disease in the joint or adjacent bone. Both patients were treated initially with analgesics because it was thought to be due to a musculoskeletal condition. In both cases subsequent phlebography showed extensive occlusive thrombosis of the calf, popliteal, and femoral veins. In addition, case 2 had occlusive thrombosis of both saphenous veins and the iliac vein. The reason for loss of full extension is not certain, but it may be due to reflex muscle spasm. It is of interest that in both cases the limitation of extension was present when the patient was first seen by his doctor.

The clinical signs and tests used in the diagnosis of deep vein thrombosis have recently been reviewed. ${ }^{1}$ Muscle spasm and irritability of the adductors and gastrocnemii have been recorded, ${ }^{2}$ as has sciatic neuritis from pelvic vein thrombosis. ${ }^{3}$ This sign would appear to be a rare presentation of extensive deep vein thrombosis with or without superficial thrombosis and should be kept in mind in the presence of a flexed knee of recent onset where no joint lesion is found.-We are, etc.,

T. P. Corrigan

Department of Surgery

King's College Hospital Medical School.

1 Shafer, N., and Duboff, S., Angiology, 1971, 22,

2 De Takats, G., Vascular Surgery. Philadelphia, Aird, I., Companion in Surgical Studies, 2nd
edn. Edinburgh and London, Livingstone, 1957.

\section{Preserving Urinary Glucose for Estimation}

SIR,-When involved in research in the endocrine unit at this hospital some years ago we became interested in the preservation of glucose in urine for estimation. Unine samples from patients received in the laboratory both within 12 hours of voiding and, in error, after several days of waiting at ambient temperatures, occasionally showed obvious infection. We doubted the efficacy of toluene, which was then in vogue, as a preserving agent. It also interfered with pipetting. We therefore studied the problem as follows.

Three specimens of urine, two turbid and one clear, known to be infected (Escherichia coli, Proteus mirabilis, and $P$. rettgeri) were obtained. After each sample was shown to give a negative Clinistix reaction dextrose was added to a concentration of about $100 \mathrm{mg} / 100 \mathrm{ml}$ urine. Each sample was then split into four $50-\mathrm{ml}$ parts, which were treated as follows: (1) control urine withou added preservative; (2) urine + solid benzoic acid; (3) urine + a complete or incomplete layer of toluene; and (4) urine + chlorhexidine diacetate (Hibitane) to produce a final concentration of $1 / 2,000$. The twelve urine samples were left on the bench and serial estimations of reducing substance ("glucose") were carried out on each over the next few weeks, using the Harding modification of the Schaffer-Hartmann method. 1

It was found that in all but the three chlorhexidine-preserved samples the concentration of urinary glucose fell, reaching about $30 \mathrm{mg} / 100 \mathrm{ml}$ within 24-72 hours. Chlorhexidine did not appear to interfere with the method of glucose estimation but sometimes precipitated in the presence of urinary phosphates. This did not appear to affect its antibacterial properties. Since this study, wards have been supplied with bottles for urinary glucose collections containing $0.25 \mathrm{~g}$ of chlorhexidine diacetate powder per expected $500 \mathrm{ml}$ urine.

We concluded that neither toluene nor benzoic acid was effective as a preservative of glucose in infected urine kept at room temperatures, but chlorhexidine was satisfactory. However, information reaching us has indicated that toluene continues to be used as an agent to prevent degradation of glucose and other substrates such as uric acid. It may be useful to remind people that this cannot be relied upon.

We thank Dr. Dutton of the bacteriology department, Hammersmith Hospital, for advice and Dr. D. G. Higgins, of Imperial Chemical Industries Ltd., for the generous supply of chlorhexidine diacetate used in this study.

-We are, etc.

G. E. Lovat

Department of Medicine,

Hammersmith Hospital,

B. Coates

1 King, E. J., and Wootton, I. D. P., Micro analysis in Medical Biochemistry, 3rd edn., p.

\section{Prevention of Deep Vein Thrombosis}

SIR,-Deep venous thrombosis with consequent massive pulmonary embolism still remains a potent cause of postoperative death. It is alleged that the majority of these thromboses are initiated during the operative period, when Virchow's triad of venous stasis, damage to the vein wall, and increased blood coagulability are maximal. The leg is the most usual site for the thrombosis of the deep veins to occur, and there is evidence that the soleal plexus of veins is especially liable as both venous stasis and damage to the vein wall will occur if the calves of the unconscious patient are compressed by the weight of his legs during a lengthy operation. Thrombosis in this site may propagate into the larger leg veins and eventually result in massive embolism. In order to reduce the risk of soleal vein thrombosis it is current practice to place a rubber pad under both Achilles tendons while the patient is on the operating table, so relieving pressure on the calves.

However, after major surgery these days the patient is removed from the operating table on to a firm bed, the pad under his heels is removed, and he is transferred to the recovery room, where he may remain for some hours before regaining full consciousness and even then be reluctant to move his legs because of pain. During this period his calves are pressed firmly down on to the mattress not only by the weight of his legs but also by the weight of the bedclothes.

It is currently my practice to insert a plastic foam pad $18 \times 3 \times 3$ in $(46 \times 7.6 \times 7.6$ $\mathrm{cm}$ ) in size beneath the Achilles tendons of patients who are deeply unconscious or who have had major surgery on transfer from the operating table to their bed. This pad travels with the patient to the ward and remains in place until he kicks it away. The pad can be cut from a sheet of plastic foam, may be used many times, and costs about $10 \mathrm{p}$. Its use is being adopted by other surgeons in this hospital and also in the intensive care unit. Much has been written recently on prophylactic measures against deep vein thrombosis, but little consideration has been given to the immediate postoperative period and I hope the simple, and possibly effective, technique advocated here will merit adoption.-I am, etc.

\section{JULIAN NEELY}

Crawley Hospital,

\section{Autoimmunity in Childhood Pulmonary} Alveolar Proteinosis

SIR,-In your leading article on pulmonary alveolar proteinosis (12 February 1972, p. 395) you drew attention to Gough's suggestion, based on animal experiments, that the production of the characteristic alveolar reaction found in this disease might depend on an altered state of immunity. Clinical support for this theory was shown by Colón et al..$^{2}$ who, in a review of 23 cases of ohildhood alveolar proteinosis, found a high incidence of lymphopenia, low immunoglobulin levels, and thymic alymphoplasia. I wish to present the 24th case of alveolar proteinosis in childhood, in which there was not only a low serum IgA level, but also 
autoantibody to smooth muscle antigen (S.M.A.). Autoantibodies have not previously been reported in this disease.

A $3 \frac{1}{2}$-year-old girl was admitted to hospital with a six-months history of cough, exertional dyspnoea and cyanosis, and severe weight loss. On examination she was apyrexial, emaciated, and had marked intercostal indrawing but no added breath sounds. Chest $x$-rays showed a fine general mottling of both lung fields. Repeated sputum cultures for tubercle bacilli were negative, as was the Mantoux test. The haematological findings, including differential white cell counts and blood and bone marrow films, were normal. The serum aspartate aminotransferase (SGOT) level (54 units) was slightly elevated for the laboratory technique used and, on one occasion only, she had a raised alkaline phosphatase level (62 King-Armstrong units). All the other liver function tests were normal. Paper electrophoresis of the serum showed only a slightly raised $\alpha_{3}$-globulin level. However, estimation of the individual immunoglobulin levels showed a low IgA (<40 mg/100 ml) with normal IgM (248 mg/ $100 \mathrm{ml})$ and $\mathrm{IgG}(920 \mathrm{mg} / 100 \mathrm{ml})$ levels. Her serum was screened for autoantibodies by the recommended immunofluorescence technique ${ }^{3}$ and there was a strong reaction" $(+++)$ with S.M.A. A lung biopsy showed the classic histological appearance of alveolar proteinosis. Immunofluorescence studies of frozen tissue showed no abnormal deposition of immunoglobulins in the lung, nor could autoreactive lung-specific antibodies be demonstrated in her serum. Despite repeated bronchial lavage her condition deteriorated rapidly and she died 10 months after the onset of the illness. Permission for necropsy was refused.

This case demonstrates the two important (and possibly related) differences between the adult and childhood forms of alveolar proteinosis: (1) In children the disease has a strikingly more serious prognosis. Of the 23 patients reviewed by Colón, the majority died in less than one year, and by the second year the mortality was $100 \%$. In Davidson's review of 139 patients (135 of whom were adults) only 38 died from alveolar proteinosis or its complications, many living for several years and 34 recovering spontaneously. (2) In children the disease is associated with immunological abnormalities. In Davidson's comprehensive review of the adults no immunological abnormalities are reported, whereas in at least 13 out of the 24 childhood cases reported there is evidence of disordered immunity.

Interestingly, a low serum IgA level was the most consistent immunoglobulin $a b-$ normality found by Colón. As $\operatorname{IgA}$ is responsible for the local protection of muoussecreting epithelial surfaces it is possible that these children have a lowered resistance to a normally innocuours organism, possibly a virus, which provokes the accumulation of pneumocytes and the excessive surfactant production which characterizes alveolar proteinosis.

Low serum IgA levels are also found in some cases of coeliac disease. ${ }^{5}$ Though no association between coeliac disease and alveolar proteinosis has been reported, in the majority of childhood cases there is very severe weight loss, usually associated with chronic diarrhoea. These symptoms are infrequent in the adult cases.

The presence of antibody to S.M.A. is particularly interesting in view of the recent work by Farrow et al. ${ }^{6}$ on the nature of S.M.A. They have shown it to be situated on microfilaments just beneath the surface membrane of certain cells. They suggest that the presence of serum antibodies to S.M.A in patients with liver disease is caused by viral damage to the liver cell exposing the microfilaments, thus allowing the (S.M.A.) antigen to become immunogenic. Since these mirofilaments have been observed in fetal lung tissue, it is possible that a similar pro cess could induce the production of S.M.A. in some lung diseases. In support of this such antibodies have been demonstrated in two patients with idiopathic pulmonary disease. ${ }^{78}$

The two-year mortality in children with alveolar proteinosis is $100 \%$ and in adult it is less than $20 \%$. It is possible that this is due to the differing immunological status of the two populations, that of the affected children being abnormal.-I am etc.

Department of Pathology,

Elizabeth S. Gray

1 Gough, J., British Medical Fournal, 1967, 1, 629. Colón, A. R., Lawrence, R. D., Mills, S. D.. and 'Connell, E. J., American

of Children, 1971, 121, 48

Beck, J. S., Association of Clinical Pathologists Broadsheet, 1971, no. 69.

Davidson, J. M., and Macleod, W. M., British Fourmal of Diseases of the Chest, 1969, 63, 13.
Crabbé, P. A., and Heremans, J. F., American fournal of Medicine, 1967, 42, 319. W. L. J., Holborow, E. J., and Brighto Lancaster Smith, M. J., Benson, M. K., and Strickland, I. D., Lancet, 1971, 1, 473. Mason, A. M. S., McIllmurray, M. B., Golding,
P. L., and Hughes, D. T. D., British Medical fournal, 1970, 4, 596 .

\section{Cancer and the Patient}

SIR,-I greatly enjoyed and endorse $\mathrm{Mr}$. Charles Wright's Personal View (6 October, p. 45) that, as in many other social situations and problems, we lag behind the United States in free, uninhibited communication. I am persuaded also that a principal barrier to good doctor/patient relations in Britain is often a lamentable failure on the part of doctors to take the time and trouble to explain in a humane and intelligible way what is wrong, what is to be done, and what it is likely that the future holds.

I have, nonetheless, a slight reservation regarding cancer. This frightening, primitive word seems to have inbuilt such a nightmarish image that many patients are unable to endure it, and so there are cases in which a tactful little charade is played out by doctor, parient, and family, all well aware that the illness is cancer, yet managing to diminish emotional suffering by refraining from referring to it in direct terms. It is ? pity that we do not have readily available ? less horrifying name; maybe "prolifera" would do.-I am, etc.,

Department of Pathology,

W. ARTHUR MACKEY

Royal Infirmary
Glasgow

SIR,-I was very interested in Mr. Charles Wright's Personal View (6 October, p. 45) and in the ensuing correspondence. During the whole of my active professional life I was particularly interested in the management of cancer-in my case, of the ear, nose, and throat-and for 25 years conducted a combined follow up clinic with my radiotherapy colleague. This long association enabled us to make an accurate long term assessment of the patient's reactions to his disease and its treatment.

I would entirely agree with $\mathrm{Mr}$. R. M. Kirk (20 October, p. 164) that, at any rate up till now, the vast majority of British patients do not wish to be told the diagnosis, and this attitute was well demonstrated over the years in the combined follow-up clinic. To anyone but the most unintelligent radioherapy means only one thing, but nothing was ever said for some five or six years, after which a number of patients discussed the matter quite openly. By that time they realized that their chances of permanent recovery were good. In a clinic like ours the doctor/patient relationship was very close and friendly and I cannot remember any instance of a patient suggesting that he should have been told exactly what was the matter with him at the start.

My policy therefore was not to tell the patient unless he asked, and this I taught my students. On the other hand I also emphasized that, if a patient put the direct question, he must be told the truth. To lie to a patient is quite unjustifiable and morally wrong. It will destroy the confidence of both patient and relatives, though a slightly optimistic view of the prognosis could in some cases be permissible. I am surprised at the suggestion that British consultants habitually lie to their cancer patients, and do not believe that this is so. In my experience "going to pieces" very rarely occurred. There were two suicides in some 300 cases. One was a patient who had asked and been told the diagnosis and the other one who had not.

I am not submitting that the North American custom may not, in the long run, be right, but it will take the time to become accepted in this country and it would be a dangerous mistake to adopt it too suddenly.-I am, etc.,

Kingsbridge, Devon

J. W. S. LINDAHL

\section{Muscosal Ulceration in Coeliac Disease}

SIR,-We note the comments of Drs. P. F. Fitzpatrick and A. G. MacIver (1 September, p. 502) on our paper (28 July, p. 212). The diagnosis of coeliac disease was made in our patient as subtotal villous atrophy was present in two separate jejunal biopsy specimens taken at sites different from the ulcerated areas.

The differentiation of ooliac disease with mucosal uloeration from ulcerative jejunitis may indeed be difficult; other authors have classified the same case in different ways. ${ }^{12}$ We believe the term ulcerative ieiunitis should include those cases in which the villous architecture of the jejunum at sites away from the ulcerated areas is normal; however, when subtotal villous atrophy is present a diagnosis of coeliac disease with ulceration should be made. There will remain cases in which the non-ulcerated jejunal mucosa shows partial villous atrophy or only patchy villous changes; the classificarion of these cases is difficult. A gluren challenge after healing has occurred may be of helo, but in our patient we were unwilling to carry this out in view of the severity of the initial illness. With regard to the failure to respond to gluten withdrawal, this has been noted previously in coeliac disease with $^{2}$ and without ${ }^{3}$ muoosal ulceration. Not only may the addition of corticosteroids initiate a response, ${ }^{3}$ but corticosteroids alone may produce partial or complete healing."

The possibility of Crohn's disease was considered, but despite an extensive search 